\title{
Missed Opportunities for Treatment of Opioid Use Disorder in the Hospital Setting: Updating an Outdated Policy
}

\author{
Justin Berk, MD, MPH, MBA ${ }^{1 *}$, Kendall M Rogers, $\mathrm{MD}^{2}$, Deanna J Wilson, MD, MPH ${ }^{3}$, \\ Ashish Thakrar, $\mathrm{MD}^{4}$, Leonard Feldman, $\mathrm{MD}^{5}$
}

\begin{abstract}
${ }^{1}$ Departments of Pediatrics and Medicine, Warren Alpert School of Medicine at Brown University, Providence, Rhode Island; ${ }^{2}$ Department of Medicine, New Mexico University Health Sciences Center, Albuquerque, New Mexico; ${ }^{3}$ Departments of Medicine and Pediatrics, University of Pittsburgh, Pittsburgh, Pennsylvania; ${ }^{4}$ Department of Medicine, Johns Hopkins Hospital, Baltimore, Maryland; ${ }^{5}$ Departments of Medicine and Pediatrics, Johns Hopkins Hospital, Baltimore, Maryland.
\end{abstract}

\section{THE PROBLEM AND THE ROLE OF THE HOSPITALIST}

Opioid use disorder (OUD) is a common, underrecognized, undertreated, and deadly medical condition. Although the focus of addressing the opioid epidemic has been centered in the outpatient setting, hospitalists play an important-and often underutilized-role in identifying OUD, initiating treatment, and assisting with linkage to longitudinal care after discharge.

Over the past 20 years, the annual rate of hospital discharges documenting OUD has quadrupled.' During 2010-2016, the annual discharge rate for heroin overdoses increased by $23 \% .^{1}$ Although the total number of hospitalizations in the United States remained stable from 2002 to 2012, the number of admissions for opioid abuse or dependence increased from 301,707 to 520,275 . More than 500,000 hospital admissions per year ( $1 \%$ of total nationwide hospitalizations) are now due primarily to OUD. ${ }^{2}$

Injection opioid use increases the risk of endocarditis, osteomyelitis, septic arthritis, and epidural abscesses, conditions that often prolong hospitalizations and frequently lead to readmissions. Admissions for OUD-related infections are rising at a startling rate. Between 2002 and 2012, the number of admissions for infections associated with OUDs had increased from 3,421 to $6,535 .{ }^{2}$ In addition to providing the opportunity to diagnose OUD, hospitalizations offer an ideal time to engage patients in OUD treatment and linkage to outpatient care.

Although we uniformly offer patients antibiotic treatment for acute infection, hospitalists should consistently incorporate treatment of OUD to address the root cause of these admissions. As infection is but one sequela of the underlying disease of addiction, treating without medications for OUD (MOUD) would be akin to treating a diabetic foot ulcer with antibiotics and not providing medications to improve glycemic control. Omitting such addiction treatment can contribute to treatment failure and worse health outcomes. Among patients with

*Corresponding Author: Justin Berk, MD, MPH, MBA; Email: jberk1@lifespan. org.

Published online first December 18, 2019.

Received: July 10, 2019; Revised: November 4, 2019;

Accepted: November 5, 2019

๑ 2020 Society of Hospital Medicine DOI 10.12788/jhm.3352 endocarditis and an associated valve repair, those who continue injection drug use have a 10 times higher risk of death or reoperation between 90 and 180 days after repair than those not engaged in drug use. ${ }^{3}$

Despite data demonstrating the significant benefit and the minimal harm of MOUD, significant gaps remain in providing MOUD and linking patients from the hospital to community care. ${ }^{1,4}$ Hospital encounters are missed opportunities to provide life-saving MOUD treatment; the majority of patients with OUD do not receive evidence-based treatment while inpatients. ${ }^{5}$ Rosenthal et al. found that of 102 patients admitted with injection drug use-associated infective endocarditis from 2004 to 2014 , only $8 \%$ received MOUD, and approximately half had a documentation of substance use treatment in their discharge worksheet. ${ }^{4}$ In Massachusetts, among individuals who experienced a nonfatal opioid overdose and had interaction with healthcare services, only $26 \%$ were on MOUD 1 year later. ${ }^{6}$ Based on our experience, a substantial proportion of patients with OUD do not seek or have access to medical care; acute care settings offer a critical opportunity to engage them in treatment for their addiction.

\section{WHY SHOULD HOSPITALISTS INITIATE BUPRENORPHINE?}

First, buprenorphine effectively treats withdrawal symptoms. Buprenorphine and methadone are superior to other medications in treating symptoms of withdrawal.? If withdrawal symptoms are treated, patients are less likely to leave against medical advice ${ }^{8}$ and are more likely to complete treatment.

Second, MOUD is the standard of care for treating OUD.? Medications include the full agonist methadone, the partial agonist buprenorphine, and the long-acting antagonist naltrexone. Although all these drugs are effective and legal to initiate for inpatients, ${ }^{6}$ this perspective focuses on buprenorphine in an effort to draw attention to associated policy barriers. Buprenorphine is the only MOUD that can be offered as office-based therapy by providers in the outpatient setting. Meta-analyses show that MOUD is associated with lower rates of mortality, illicit opioid use, HIV transmission, and violent crime and arrest. ${ }^{9}$

Third, MOUD treatment, rather than just referral, leads to higher long-term treatment success. ${ }^{10}$ When initiating buprenorphine in the hospital, treatment retention rates at 1 month were double that of referral alone. Six months after discharge, patients were 
five times more likely to remain engaged in treatment compared with those who received a detoxification protocol only.

Fourth, buprenorphine is not only effective, but it is also safe and has low risks of misuse. Because buprenorphine is a partial agonist, it has both a ceiling effect on respiratory depression (decreasing potential lethality) and on euphoria (decreasing the likelihood of misuse). Among individuals who took nonprescribed buprenorphine on the street, less than 7\% reported taking it for any attempt at euphoria. Instead, people with OUD most often use nonprescribed or diverted buprenorphine to treat withdrawal symptoms. ${ }^{11}$

Fifth, buprenorphine treatment is associated with fewer hospital readmissions. ${ }^{12}$

Finally, initiating OUD treatment is feasible in the hospital setting. Any hospitalist can legally prescribe buprenorphine to treat opioid withdrawal for hospitalized patients admitted for medical or surgical reasons. A waiver is necessary only for prescribing at the time of hospital discharge for use in non-inpatient settings of care.

\section{A POLICY BARRIER: THE X WAIVER}

The United States Congress passed the Drug Addiction Treatment Act (DATA) of 2000, which codified the $X$ waiver, in response to the growing opioid crisis. Only those providers with the DATA $X$ waiver can write buprenorphine prescriptions to be filled in an outpatient pharmacy. To obtain an $X$ waiver, physicians must complete an 8-hour course, whereas physician assistants and nurse practitioners must complete a 24-hour course. This training far exceeds any required training to prescribe opioids for pain.

Unfortunately, the $X$ waiver requirement obstructs hospitalists from initiating buprenorphine in the inpatient setting in the following ways: (1) hospitalists often choose not to initiate chronic buprenorphine treatment if they lack the $X$ waiver that would allow them to write the discharge prescription and/or (2) they are unable to identify a waivered provider in the community to continue the prescription. Unfortunately, only $6 \%$ of all medical practitioners are waivered to prescribe buprenorphine; greater than $40 \%$ of US counties are "buprenorphine deserts," with no providers waivered to prescribe buprenorphine. ${ }^{13}$

\section{A POLICY PROPOSAL}

To address the opioid crisis, we must rethink our current policies. The Department of Health and Human Services should eliminate the $X$ waiver and allow any licensed physician, nurse practitioner, or physician assistant to prescribe buprenorphine. ${ }^{14}$ Recent American Medical Association Opioid Task Force recommendations have called to "remove... inappropriate administrative burdens or barriers that delay or deny care for FDA-approved medications used as part of medicationassisted treatment for OUD." ${ }^{15}$ Legislation to remove the $X$ waiver has been proposed in the United States. ${ }^{16}$

The removal of a buprenorphine waiver requirement has had success in other settings. The French deregulation of buprenorphine was associated with a reduction in opioid overdose deaths by $79 \%$. Similar success in the United States would save an estimated 30,000 lives yearly. ${ }^{14}$ Removing the $X$ waiver is an important step in empowering hospitalists to initiate MOUD for individuals in the hospital setting. Moreover, it opens the door to more outpatient primary care providers serving as community linkages for long-term addiction care.

\section{NOT A PANACEA}

Without the $X$ waiver, the associated OUD training will no longer be required. This could have unintended consequences. For example, if hospitalists order buprenorphine while opioids remain active, precipitated withdrawal may ensue. Crucially, the current literature does not indicate that the required $X$ waiver training improves knowledge, patient care, or outcomes. ${ }^{17}$ Nevertheless, MOUD and addiction training may help reduce knowledge gaps and empower providers to engage in productive conversations surrounding addiction. This highlights the crucial role of physician organizations, such as the Society of Hospital Medicine, in educating hospitalists about MOUD. (This organization, among others, has developed robust MOUD training. ${ }^{18}$ )

It is also important to acknowledge that the waiver is only one obstacle. Other barriers have been identified in initiating buprenorphine, including access to treatment after discharge, access to social work support, and lack of EMR order sets, among others. ${ }^{19}$ Professional societies, hospitals, and hospitalists need to help address these barriers through ancillary support staff, quality improvement initiatives, and improved inpatient treatment of withdrawal with MOUD. This can be done successfully; one study found that $82 \%$ of hospitalized patients who engaged in a new transitional opioid program subsequently presented to outpatient opioid treatment. ${ }^{20}$ Novel interventions must be part of a hospital-wide approach to optimizing improved longitudinal treatment for patients suffering from addiction.

\section{CONCLUSION}

Hospitalization is an ideal opportunity for clinicians to diagnose and treat OUD in a population that often has not sought, or has fallen out of, addiction treatment. Hospitalists can and should initiate buprenorphine in appropriate inpatients and plan for their transition to chronic care. Eliminating the waiver in combination with designing innovative educational opportunities and systems approaches to provide better linkages to outpatient OUD treatment is needed to combat the opioid crisis. To enable more hospitalists to successfully initiate long-term buprenorphine therapy-and to enable more outpatient providers to continue prescriptions - we must eliminate the $X$ waiver.

Disclosures: Dr Wilson received honorarium from the American Society of Addiction Medicine for teaching and creating CME outside the submitted work. All other authors have no conflicts of interest and have received no related funding for this topic.

\section{References}

1. Peterson C, Xu L, Florence C, Mack KA. Opioid-related US hospital discharges by type, 1993-2016. J Subst Abuse Treat. 2019;103:9-13. https://doi. org/10.1016/j.jsat.2019.05.003 
2. Ronan MV, Herzig SJ. Hospitalizations related to opioid abuse/dependence and associated serious infections increased sharply, 2002-12. Health Aff. 2016;35(5):832-837. https://doi.org/10.1377/hlthaff.2015.1424

3. Shrestha NK, Jue J, Hussain ST, et al. Injection drug use and outcomes after surgical intervention for infective endocarditis. Ann Thorac Surg. 2015;100(3):875-882. https://doi.org/10.1016/j.athoracsur.2015.03.019

4. Rosenthal ES, Karchmer AW, Theisen-Toupal J, Castillo RA, Rowley CF Suboptimal addiction interventions for patients hospitalized with injection drug use-associated infective endocarditis. Am J Med. 2016;129(5):481-485. https://doi.org/10.1016/j.amjmed.2015.09.024

5. Winetsky D, Weinrieb RM, Perrone J. Expanding treatment opportunities for hospitalized patients with opioid use disorders. J Hosp Med. 2017;13(1):62 64. https://doi.org/10.12788/jhm.2861

6. Larochelle MR, Bernson D, Land T, et al. Medication for opioid use disorder after nonfatal opioid overdose and association with mortality: a cohort study. Ann Intern Med. 2018;169(3):137-145. https://doi.org/10.7326/M17-3107

7. Gowing L, Ali R, White JM, Mbewe D. Buprenorphine for managing opioid withdrawal. Cochrane Database Syst Rev. 2017;(2):CD002025. https://doi. org/10.1002/14651858.CD002025.pub5

8. Ti L, Ti L. Leaving the hospital against medical advice among people who use illicit drugs: a systematic review. Am J Public Health. 2015;105(12):e53-e59. https://doi.org/10.2105/AJPH.2015.302885

9. Schuckit MA. Treatment of opioid-use disorders. $N$ Engl J Med. 2016;375(4):357-368. https://doi.org/10.1056/NEJMra1604339

10. Liebschutz JM, Crooks D, Herman D, et al. Buprenorphine treatment for hospitalized, opioid-dependent patients. JAMA Intern Med. 2014;174(8):1369. https://doi.org/10.1001/jamainternmed.2014.2556

11. Cicero TJ, Ellis MS, Surratt HL, Kurtz SP. Factors contributing to the rise of buprenorphine misuse: 2008-2013. Drug Alcohol Depend. 2014;142:98-104 https://doi.org/10.1016/j.drugalcdep.2014.06.005
12. Moreno JL, Wakeman SE, Duprey MS, Roberts RJ, Jacobson JS, Devlin JW. Predictors for 30-day and 90-day hospital readmission among patients with opioid use disorder. J Addict Med. 2019;13(4):306-313. https://doi. org/10.1097/ADM.0000000000000499

13. Andrilla CHA, Moore TE, Patterson DG, Larson EH. Geographic distribution of providers with a DEA waiver to prescribe buprenorphine for the treatment of opioid use disorder: a 5-year update. J Rural Heal. 2019;35(1):108-112. https://doi.org/10.1111/jrh.12307

14. Fiscella K, Wakeman SE, Beletsky L. Buprenorphine deregulation and mainstreaming treatment for opioid use disorder: $x$ the $x$ waiver. JAMA Psychiatry. 2019;76(3):229-230. https://doi.org/10.1001/jamapsychiatry.2018.3685

15. American Medical Association Opioid Task Force. AMA Opioid Task Force recommendations offer roadmap to policymakers American Medical Association. Accessed June 14, 2019. https://www.ama-assn.org/press-center/press-releases/ama-opioid-task-force-recommendations-offer-roadmap-policymakers

16. Tonko P. H.R.2482: Mainstreaming Addiction Treatment Act of 2019. House of Representatives (116th Congress); 2019. Accessed July 10, 2019. https:// www.congress.gov/bill/116th-congress/house-bill/2482

17. Frank JW, Wakeman SE, Gordon AJ. No end to the crisis without an end to the waiver. Subst Abus. 2018;39(3):263-265. https://doi.org/10.1080/0889707 7.2018.1543382

18. Society of Hospital Medicine. Clinical Topics: Opioid Safety. Accessed October 24, 2019. https://www.hospitalmedicine.org/clinical-topics/opioid-safety/

19. Lowenstein M, Kilaru A, Perrone J, et al. Barriers and facilitators for emergency department initiation of buprenorphine: a physician survey. Am J Emerg Med. 2019;37(9):1787-1790. https://doi.org/10.1016/j.ajem.2019.02.025

20. Shanahan CW, Beers D, Alford DP, Brigandi E, Samet JH. A transitional opioid program to engage hospitalized drug users. J Gen Intern Med. 2010;25(8):803-808. https://doi.org/10.1007/s11606-010-1311-3 